\title{
3D Digital Image Correlation Analysis of the Shrinkage Strain in Four Dual Cure Composite Cements
}

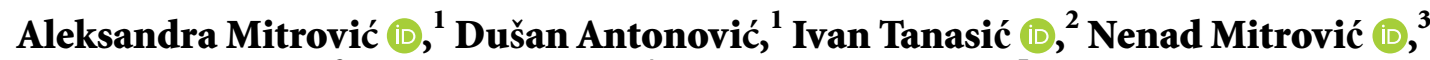 \\ Gordana Bakić $\mathbb{D}^{3}{ }^{3}$ Dejana Popović, ${ }^{4}$ and Miloš Milošević ${ }^{5}$ \\ ${ }^{1}$ University of Belgrade, Faculty of Technology and Metallurgy, Karnegijeva 4, 11000 Belgrade, Serbia \\ ${ }^{2}$ University of Belgrade, School of Dental Medicine, Rankeova 4, 11000 Belgrade, Serbia \\ ${ }^{3}$ University of Belgrade, Faculty of Mechanical Engineering, Kraljice Marije 16, 11000 Belgrade, Serbia \\ ${ }^{4}$ University of Belgrade, Institute of Nuclear Sciences Vinca, 12-14 Mike Petrovića Street, 11000 Belgrade, Serbia \\ ${ }^{5}$ Innovation Center of Faculty of Mechanical Engineering in Belgrade, Kraljice Marije 16, 11000 Belgrade, Serbia \\ Correspondence should be addressed to Ivan Tanasić; ivantanasic1@gmail.com
}

Received 25 April 2019; Revised 20 July 2019; Accepted 25 July 2019; Published 17 November 2019

Academic Editor: Nick Silikas

Copyright (c) 2019 Aleksandra Mitrović et al. This is an open access article distributed under the Creative Commons Attribution License, which permits unrestricted use, distribution, and reproduction in any medium, provided the original work is properly cited.

\begin{abstract}
The introduction of resin-based cements and an adhesive-bonding system in daily dental practice has given the opportunity to increase the retention of previously conventional cemented restorations and the optimal results in esthetic. This experimental study employed the 3D Digital Image Correlation Method (3D-DIC) for detecting shrinkage strain in four dual cured composite cements. The aim was to visualize measure, analyze, and compare strain fields in four resin-based cements using the 3D-DIC method. A total of 72 samples were divided into 4 groups considering variations in sample types, diameter, and thickness. Four types of composite cements: RelyX U200 (3 M ESPE, St. Paul, MN, USA), MaxCem Elite (Kerr, Orange, CA, USA), Multilink Automix (Ivoclar Vivadent, Schaan, Liechtenstein), and SeT PP (SDI, Australia) were used. Each type had diameters of $3 \mathrm{~mm}, 4 \mathrm{~mm}$, and $5 \mathrm{~mm}$, respectively, combined with two different values of thickness: $1 \mathrm{~mm}$ and $2 \mathrm{~mm}$. Thickness had an important role on strain detected in all tested materials showing higher strain in samples with $2 \mathrm{~mm}$ thickness compared to $1 \mathrm{~mm}$ samples. Shrinkage strain values were the highest in Set PP samples indicated the possibility of undesirable de-bonding.
\end{abstract}

\section{Introduction}

In current dental practice resin-based cements (RBCs) have usually been used for all ceramic restorations fixations, since they overcome poor mechanical, biological, and adhesion features of the previously used cement $[1,2]$. The right choice of RBCs is significant for the longevity of dental restorative materials. Composition of each ceramic system type is unique and therefore requires appropriate type of resin-based cements and cementation protocol. Following the protocol, it is important to establish adequate bond between cement and ceramic agents through adhesive or self-adhesive bonding [3-5].

Mechanical properties of the RBCs used for the cementation of all ceramics could determine long-term clinical prognosis due to adhesion problems. Improved therapy success using resin composite cements is primarily based on their significant properties [6], such as thermal and chemical stability, decreased hydrolytic degradation, better solubility, wear resistance, higher elasticity, plasticity, hardness, and strength.

The standard protocol steps prior to cementation, such as conditioning or priming pretreatments of tooth, involve using composite cements due to acidic and hydrophilic monomers and their ability to create stable chemical composition responsible for the strong bonding [7]. These monomers are also responsible for polymerization shrinkage stress and shrinkage strain, manifested during the RBCs' hardening process $[8,9]$. Additionally, curing mode and filler content have also been found to be important for strain magnitude [9].

Dual cure mode, consisting of self-cure and light cure mode, has demonstrated the mechanical properties' 


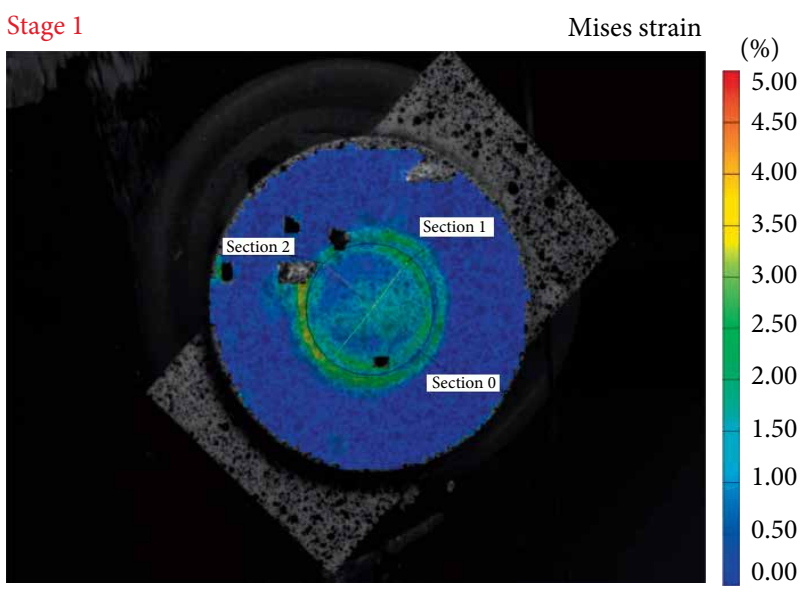

(a)

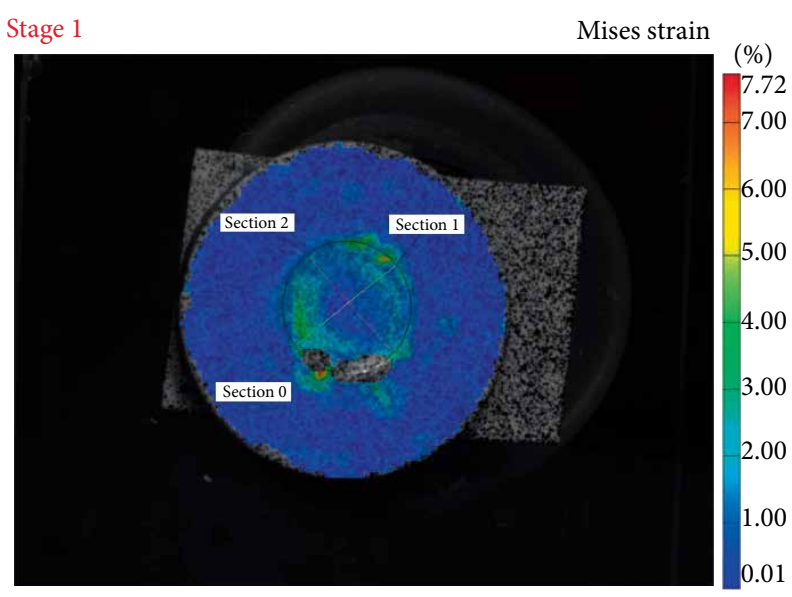

(b)

FIgURE 1: Von Mises strain field of MaxCem Elite across the sample surface showing the outer segment with higher and inner segments with lower strain values (a) sample photograph with overlaying Von Mises strain field forl mm thickness; (b) sample photograph with overlaying Von Mises strain field for $2 \mathrm{~mm}$ thickness.

enhancement. The polymerization shrinkage strain in RBCs during the hardening process can cause therapeutic failure due to de-bonding [10]. Namely, strain detected when converting monomer to polymer leads to micro-leakage and has adverse effect on longevity of restorations due to cement degradation [11-13]. In addition, the thickness of the cement layer has been found to be an important factor for the cementation [14].

We have conducted an experimental study using the $3 \mathrm{D}$ Digital Image Correlation Method (3D-DIC) as an optical technique for detecting shrinkage strain in composite cements immediately after photo-polymerization. The main goal of the study was to determine, analyze, and compare strain fields in four RBCs using the 3D-DIC method, since the shrinkage of RBCs may compromise its bonding effectiveness.

The following hypotheses of study were formulated:

(a) Strain values differ in all four tested cements.

(b) Sample thickness influences strain values.

(c) Sample diameter influences strain values.

\section{Materials and Methods}

Strain field was measured using the $3 \mathrm{D}$ optical system Aramis $2 \mathrm{M}$ (GOM, Braunschweig, Germany) based on 3D-DIC method [15]. Prior to the experiment, system calibration was performed using the calibration panel for corresponding measurement volume. The volume was chosen based on the dimensions of the measured area on the sample surface. After successful calibration, the measuring could begin.

A total of 72 samples were divided into 4 groups. The groups included 4 types of composite cements: RelyX U200 (3 M ESPE, St. Paul, MN, USA), MaxCem Elite (Kerr, Orange, CA, USA), Multilink Automix (Ivoclar Vivadent, Schaan, Liechtenstein) and SeT PP (SDI, Australia). Each type had diameters of $3 \mathrm{~mm}, 4 \mathrm{~mm}$ and $5 \mathrm{~mm}$, combined with two different values of thickness: $1 \mathrm{~mm}$ and $2 \mathrm{~mm}$. Samples were prepared by filling ring-type molds. The top surface of each sample was sprayed with fine black and white spray (Kenda Color Acrilico, Kenda Farben) to create a stochastic pattern with high contrast for image analysis. Digital images were made immediately prior and after light curing. A LED light-curing unit $\left(450-500 \mathrm{~mW} / \mathrm{cm}^{2}\right.$, LEDition, IvoclarVivadent, Schaan, Liechtenstein) was used for $40 \mathrm{~s}$ to activate polymerization. The images were then analyzed using special software (Aramis 6.2.0) to determine the von Mises strain. Analysis of the von Misses strain fields was done using three sections (Sections 0, 1 and 2). Section 0 is a circular section positioned on the material-mold interface of the sample. Sections 1 and 2 are linear sections positioned orthogonally to each other. Diameter of the Section 0 and length of the Sections 1 and 2 correspond to sample diameter.

All tested materials are dual cure. MaxCem Elite and SeT PP are self-adhesive and self-etch cements. Light-curing mode was used. The experiments were done at room temperature.

The obtained data were statistically analyzed using the general linear model (GLM) for factors "material", "diameter" and "thickness", as well as their interactions. When the interaction was significant $(p<0.05)$, one-way analysis of variance (ANOVA) was performed with Tukey's post-hoc tests and Bonferroni correction. The level of significance was set at $p=0.05$. Statistical analysis was performed using the software package Minitab 16 (Minitab Inc., State College, PA, USA).

\section{Results}

Figures 1-4 are representative images of the von Mises strain across the surface of each material with diameter $5 \mathrm{~mm}$ after photo-polymerization. Maximum von Misses strain values were measured in Section 0 in all figures. As seen in Figures 1-4, all composite cements sized $\emptyset 5 \times 2 \mathrm{~mm}$ expressed higher strain values in Section 0 compared to $\varnothing 5 \times 1 \mathrm{~mm}$ samples. Furthermore, MaxCem Elite and Multilink Automix samples $\varnothing 5 \times 1 \mathrm{~mm}$ showed a similar distribution of the highest strain 


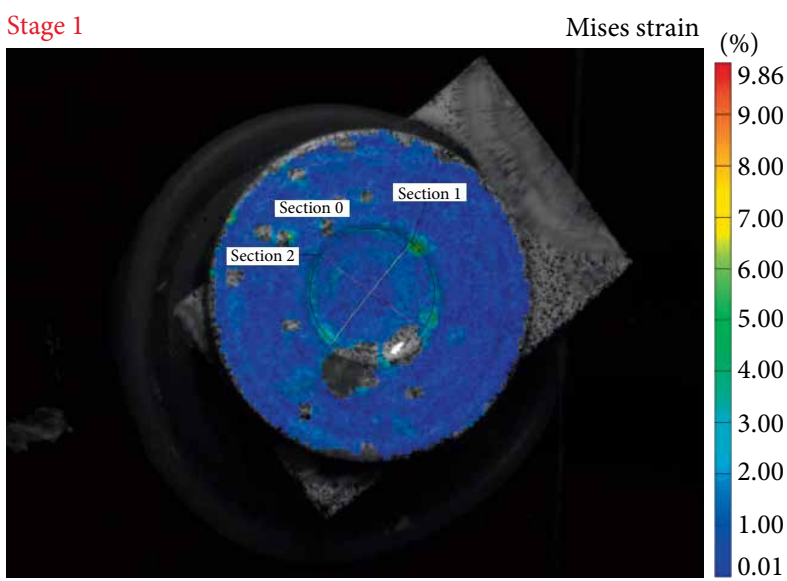

(a)

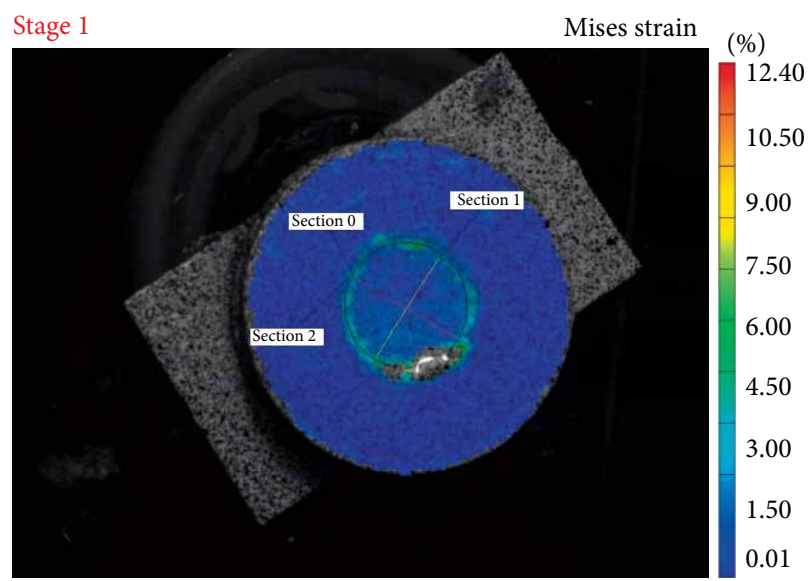

(b)

Figure 2: Von Mises strain field of SeT PP across the sample surface showing the outer segment with higher and inner segments with lower strain values (a) sample photograph with overlaying Von Mises strain field for $1 \mathrm{~mm}$ thickness; (b) sample photograph with overlaying Von Mises strain field for $2 \mathrm{~mm}$ thickness.

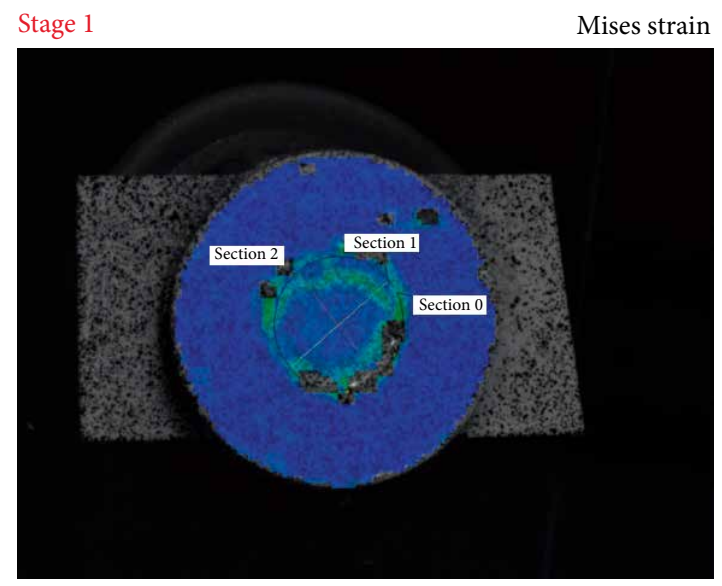

(a)

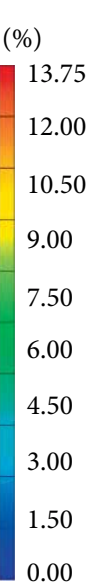

0.00

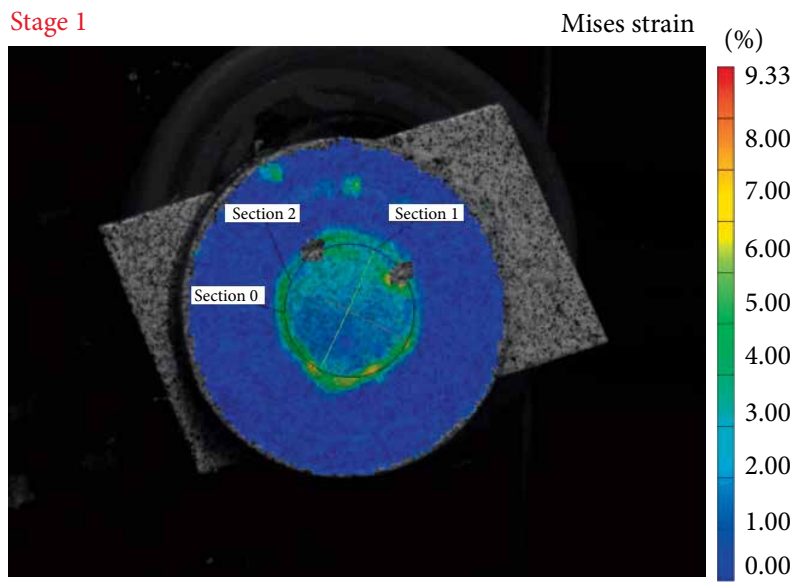

(b)

FIGURE 3: Von Mises strain field of Relyx U200 across the sample surface showing the outer segment with higher and inner segments with lower strain values (a) sample photograph with overlaying Von Mises strain field for $1 \mathrm{~mm}$ thickness; (b) sample photograph with overlaying Von Mises strain field for 2 mm thickness.

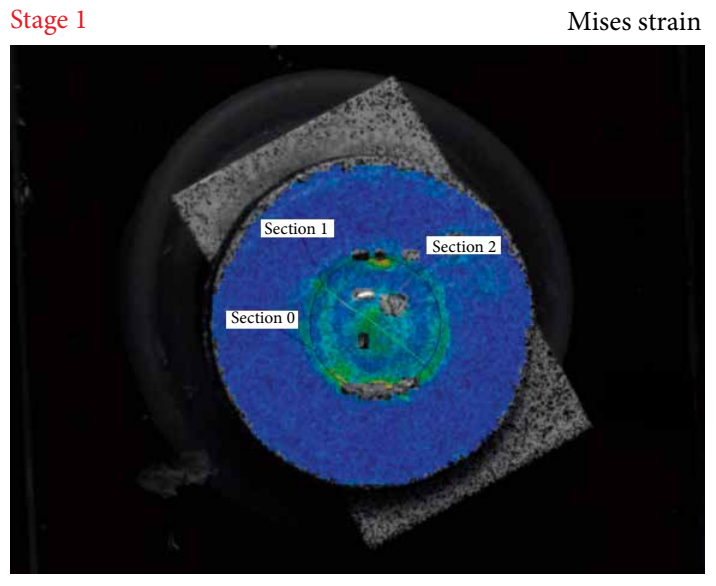

(a)

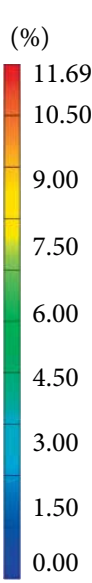

0.00

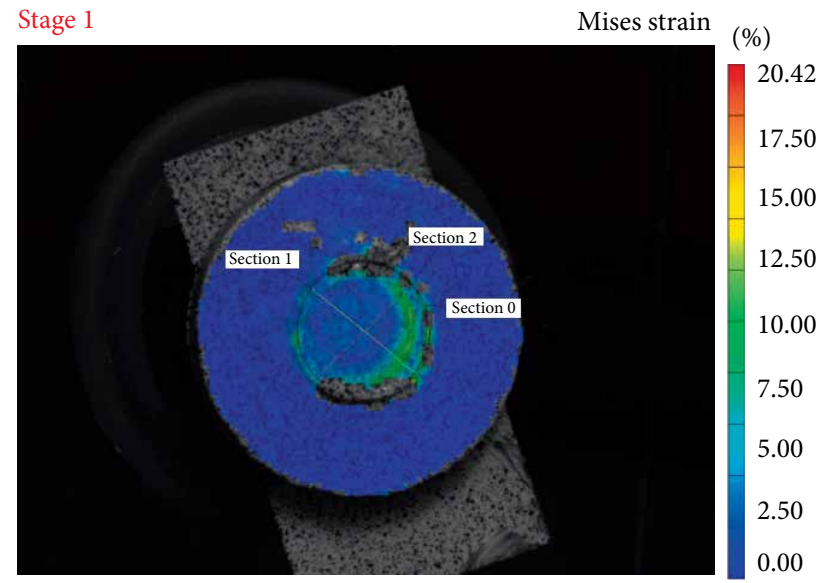

(b)

Figure 4: Von Mises strain field of Multilink Automix across the sample surface showing the outer segment with higher and inner segments with lower strain values (a) sample photograph with overlaying Von Mises strain field for $1 \mathrm{~mm}$ thickness; (b) sample photograph with overlaying Von Mises strain field for $2 \mathrm{~mm}$ thickness. 


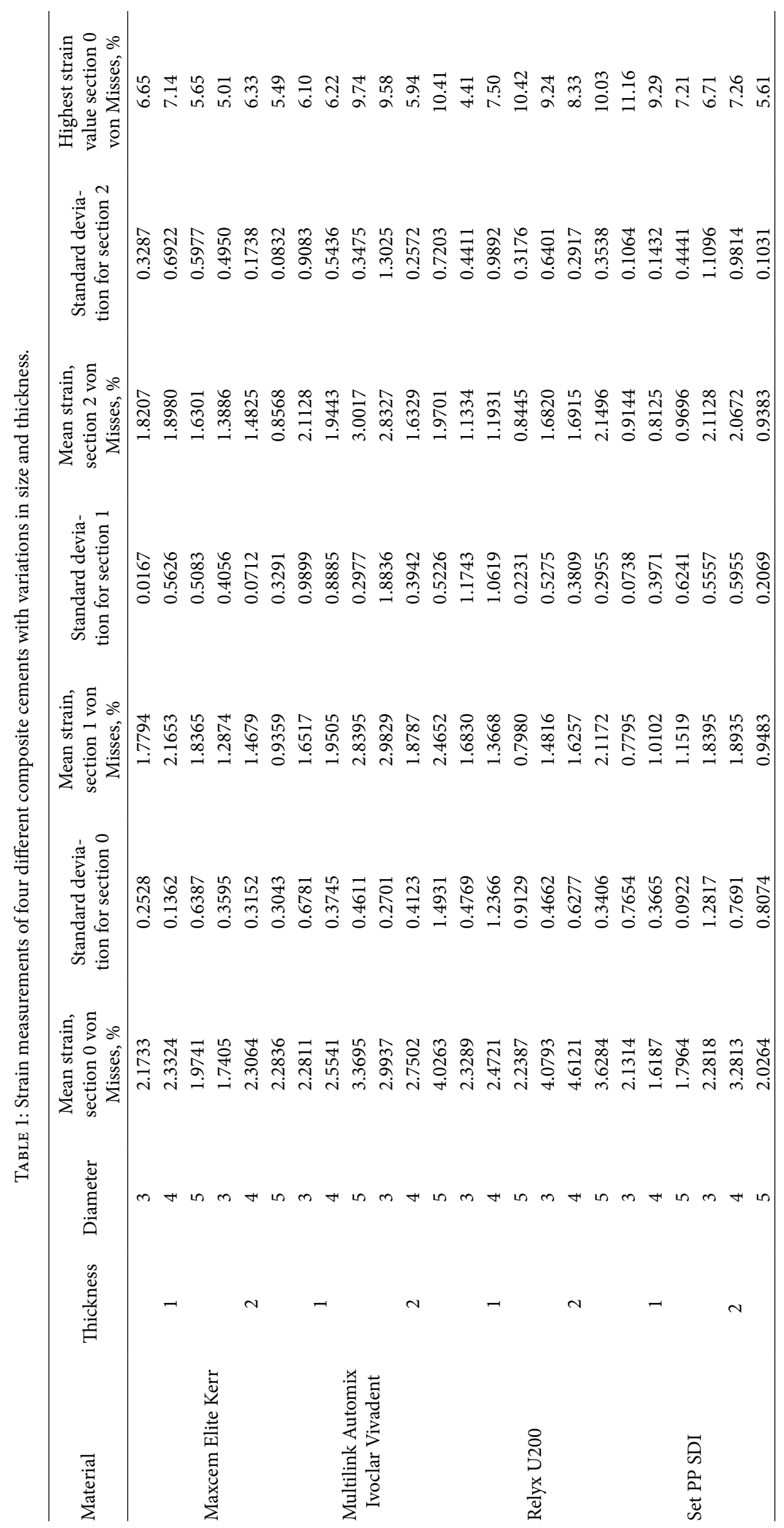


TABle 2: Analysis of Variance for Von Mises, using Adjusted SS for Tests.

\begin{tabular}{|c|c|c|c|c|c|c|c|c|c|c|}
\hline \multirow{2}{*}{ Source } & \multicolumn{2}{|c|}{$\begin{array}{l}\text { The total degrees of } \\
\text { freedom (DF) }\end{array}$} & \multicolumn{2}{|c|}{$\begin{array}{l}\text { The sequential sums } \\
\text { of squares (Seq SS) }\end{array}$} & \multicolumn{2}{|c|}{$\begin{array}{l}\text { Adjusted sums of } \\
\text { squares (Adj SS) }\end{array}$} & \multicolumn{2}{|c|}{ F-value } & \multicolumn{2}{|c|}{$\mathrm{P}$-value } \\
\hline & Section 0 & $\begin{array}{c}\text { Section } \\
1+2\end{array}$ & Section 0 & $\begin{array}{c}\text { Section } \\
1+2\end{array}$ & Section 0 & $\begin{array}{c}\text { Section } \\
1+2\end{array}$ & Section 0 & $\begin{array}{c}\text { Section } \\
1+2\end{array}$ & Section 0 & $\begin{array}{c}\text { Section } \\
1+2\end{array}$ \\
\hline Material & 3 & 3 & 16.7169 & 7.3716 & 16.7169 & 7.3716 & 5.5723 & 20.93 & 0.001 & 0.001 \\
\hline Thickness (mm) & 1 & 1 & 9.5469 & 0.8899 & 9.5469 & 0.8899 & 9.5469 & 7.58 & 0.001 & 0.007 \\
\hline Diameter (mm) & 2 & 2 & 0.7245 & 0.2412 & 0.7245 & 0.2412 & 0.3622 & 1.03 & 0.460 & 0.361 \\
\hline $\begin{array}{l}\text { Correlation } \\
\text { between material, } \\
\text { thickness and } \\
\text { diameter }\end{array}$ & 6 & 6 & 2.5884 & 2.8495 & 2.5884 & 2.8495 & 0.4314 & 4.04 & 0.476 & $0.001^{\star *}$ \\
\hline $\begin{array}{l}\text { Correlation } \\
\text { between material } \\
\text { and thickness }\end{array}$ & 3 & 3 & 7.7163 & 5.5469 & 7.7163 & 5.5469 & 2.5721 & 15.75 & $0.002^{*}$ & 0.001 \\
\hline $\begin{array}{l}\text { Correlation } \\
\text { between material } \\
\text { and diameter }\end{array}$ & 6 & 6 & 6.0954 & 1.7609 & 6.0954 & 1.7609 & 1.0159 & 2.50 & 0.058 & 0.026 \\
\hline $\begin{array}{l}\text { Correlation } \\
\text { between thickness } \\
\text { and diameter }\end{array}$ & 2 & 2 & 0.6624 & 0.4927 & 0.6624 & 0.4927 & 0.3312 & 2.10 & 0.492 & 0.127 \\
\hline Error & 48 & 120 & 22.0597 & 14.0900 & 22.0597 & & 0.4596 & & & \\
\hline Total & 71 & 143 & 66.1105 & 33.2427 & & & & & & \\
\hline
\end{tabular}

* Statistically significant correlation between cement type and diameter for the section $0,{ }^{* *}$ significant correlation between material thickness and diameter regarding sections 1 and 2 .

values in all Sections. However, Relyx U200 and SeT PP samples $\emptyset 5 \times 2 \mathrm{~mm}$ showed similar distribution of the highest strain values in all Sections.

Strain mean revealed that the von Misses strain in Section 0 , Section 1 and Section 2 did not depend on the diameter of the samples (Table 1). Samples with $2 \mathrm{~mm}$ thickness indicated significantly higher values of the von Misses strain in all Sections compared to the samples with $1 \mathrm{~mm}$ thickness. The von Mises strain was significantly higher in Section 0 than in Sections 1 and 2 for all composite cements. A significant difference regarding the thickness of the material and the type of composite cement ( $p=0.002)$ has been found (Table 2$)$. A significant difference $(p=0.001)$ in strain values has been found between the type of cement, thickness and diameter in Sections 1 and 2 (Table 2). Furthermore, ANOVA showed that with $1 \mathrm{~mm}$ thickness, Multilink Automix exhibited higher von Misses strain values compared to others. Considering samples with $1 \mathrm{~mm}$ thickness, Multilink Automix and MaxCem Elite showed significantly higher strain in Section 1 and Section 2 compared to RelyX U200 and SeT PP. RelyX U200 showed higher von Misses strain values with $2 \mathrm{~mm}$ thickness. Significantly higher values of von Misses strain were measured for Multilink Automix and RelyX U200 compared to SeT PP and MaxCem Elite in all Sections with $2 \mathrm{~mm}$ thickness.

\section{Discussion}

The study aimed to determine strain immediately after the polymerization of four composite cements in the ring type molds. It is important to emphasize that the quality of the specimen can influence the outcome of the test, as the RBCs were molded into the required test shape and then polymerized. It should be ensured that the material is adequately and uniformly cured for the appropriate amount of time and with sufficient light energy. These conditions provide the most valid test results and characterize the given material's optimal attainable properties [3].

The results presented in this study indicated nonuniform strain distribution in all tested materials. The nonuniform distribution of shrinkage strain was also reported using a single digital image/camera 2D digital image correlation, showing the highest shrinkage in subsurface parts of the sample [16]. Similarity has been found in the work of Oliveira et al. [17] who noticed increased polymerization shrinkage at the adhesive interface and possible adhesive failure. Given that intermolecular distance between the monomers is replaced by a covalent bond, polymerization shrinkage and resulting shrinkage strain are inherent to polymerization. Differences in chemical composition and filler type or size have directly influenced the shrinkage behavior. The monomer-chain mobility can be limited by the amount of the fillers, leading to decreased monomers and radical mobility, so resulting in lower shrinkage $[13,18]$. However, manufacturers do not state the precise chemical composition of all tested materials, so it is difficult to compare strain in this respect.

Previous studies $[6,10,19]$ have been conducted on the standardized sample size showing data on strain mean. Our study cannot be compared to others, as they focused on the overall volumetric shrinkage, rather than deformation fields. Using two cameras system, 3D-DIC technique provided sufficient data about out-of-plane shrinkage strain [15]. An advantage of the 3D-DIC method over other methods is the ability of full-field strain measurement [9, 20-23]. These 
methods excluded peripheral section (Section 0) although the peripheral strain has to be considered when interpreting the overall strain. 3D-DIC method additionally detected maximal von Misses strain values and determined the zones of the maximal strain through presenting images of 3D full strain field. Including several sizes of experimental samples, our study revealed additional information about sample size dependent shrinkage patterns focusing on strain field.

Increased, nonuniformly distributed shrinkage strain at adhesive interface induced by shrinkage of RBCs may compromise bonding and lead to adhesive failure. This may be of particular importance in the case of ceramic systems. Shrinkage strain values were the highest in SeT PP samples, which indicated the possibility of undesirable de-bonding. Also, finding the optimal thickness of RBC film could prevent fracture of ceramics [24-26]. De-bonding of all ceramic systems is correlated with cement thickness as a consequence of adhesion failure due to occlusal loads [27]. High shrinkage values of RBCs, as seen in the present study, may compromise their bonding effectiveness.

\section{Conclusion}

The study investigated samples of four different dual cure composite cements used in a daily dental practice, using a modern noncontact method-the 3D Digital Image Correlation. Within the limits of 3D-DIC method and the selection of tested materials, we can conclude the following: statistics showed that the von Mises strain in the peripheral zone of samples (Section 0) did not depend on the diameter of the samples, although correlation was found between thickness and peripheral zones of the samples. The two out of three hypotheses of study were confirmed. Thickness had an important impact on strain detected in all four composite cements, showing higher strain in samples with $2 \mathrm{~mm}$ thickness compared to $1 \mathrm{~mm}$ samples. The advantage of the 3D-DIC method is the ability to measure the highest strain values and to visualize the highest strain locations in tested samples. In addition, shrinkage strain values were the highest in SeT PP samples, indicating the possibility of undesirable de-bonding. Further investigation should include the role of each RBC component on the final properties of the materials and its bonding to the ceramics.

\section{Data Availability}

The von Mises results and statistical data used to support the findings of this study are included within the article. Also, the von Mises results and statistical data used to support the findings of this study are available from the corresponding author upon request.

\section{Conflicts of Interest}

The authors declare that the research was conducted in the absence of any commercial or financial relationships that could be construed as a potential conflict of interest.

\section{Authors' Contributions}

MA Crucial contribution to the article conception and design, obtaining and interpretation of the results; design of the manuscript and final revision of the manuscript being prepared for publication, as well as final approval of the version to be submitted. AD Interpretation of results; design of the manuscript and final revision of the manuscript being prepared for publication, as well as final approval of the version to be submitted. TI Contribution to the article conception and interpretation of results, drafting the article, revision of the intellectual content, as well as final approval of the version to be submitted. MN Contribution to the article conception and interpretation of results, drafting the article, revision of the intellectual content, as well as final approval of the version to be submitted. BG Obtaining of the results, contribution to the article conception and interpretation of results, revision of the intellectual content, as well as final approval of the version to be submitted. PD Obtaining of the results, contribution to the article conception and interpretation of results, revision of the intellectual content, as well as final approval of the version to be submitted. MM Obtaining and interpretation of results, revision of the intellectual content, as well as final approval of the version to be submitted.

\section{Acknowledgments}

The authors are grateful to Tam-Auto DENTAL DEPO (Novi Sad, Serbia), 3M Science Applied to Life (Belgrade, SERBIA) and Neodent (Belgrade, Serbia) for providing the materials used in this study. This research was supported by the Ministry of Education, Science and Technological Development of Republic of Serbia under Projects TR35031 and TR35040.

\section{References}

[1] A. Awada and D. Nathanson, "Mechanical properties of resin-ceramic CAD/CAM restorative materials," The Journal Prosthetic Dentistry, vol. 114, no. 4, pp. 587-593, 2014.

[2] M. Petrini, M. Ferrante, and B. Su, "Fabrication and characterization of biomimetic ceramic/polymer composite materials for dental restoration," Dental Materials, vol. 29, no. 4, pp. 375-381, 2013.

[3] N. Ilie, T. J. Hilton, S. D. Heintze et al., "Academy of dental materials guidance-resin composites: Part I-mechanical properties," Dental Materials, vol. 33, pp. 880-894, 2017.

[4] M. Fuchs, E. Gentleman, S. Shahid, R. G. Hill, and D. S. Brauer, "Therapeutic ion-releasing bioactive glass ionomer cements with improved mechanical strength and radiopacity," Frontiers in Materials, vol. 2, 2015.

[5] W. Germscheid, L. Gosse de Gorre, B. Sullivan, C. O’Neill, R. Price, and D. Labrie, "Post-curing in dental resin-based composites," Dental Materials, vol. 34, no. 9, pp. 1367-1377, 2018.

[6] J. L. Ferracane, J. W. Stansbury, and F. J. Burke, "Selfadhesive resin cements - chemistry, properties and clinical considerations," Journal of Oral Rehabilitation, vol. 38, no. 4, pp. 295-314, 2011. 
[7] K. L. Van Landuyt, Y. Yoshida, I. Hirata et al., "Influence of the chemical structure of functional monomers on their adhesive performance," Journal of Dental Research, vol. 87, no. 8, pp. 757-761, 2008.

[8] J. B. C. Meira, R. R. Braga, R. Y. Ballester, C. B. Tanaka, and A. Versluis, "Understanding contradicting data in contraction stress tests," Journal of Dental Research, vol. 903, no. 3, pp. 365-370, 2011.

[9] A. Amirouche-Korichi, M. Mouzali, and D. C. Watts, "Effects of monomer ratios and highly radiopaque fillers on degree of conversion and shrinkage-strain of dental resin-composites," Dental Materials, vol. 25, no. 11, pp. 1411-1418, 2009.

[10] T. Spinell, A. Schedlea, and D. C. Watts, "Polymerization shrinkage kinetics of dimethacrylate resin-cements," Dental Materials, vol. 25, no. 8, pp. 1058-1066, 2009.

[11] M. C. Cagidiaco, C. Goracci, F. Garcia-Godoy, and M. Ferrari, "Clinical studies of fiber posts. a literature review," International Journal of Prosthodontics, vol. 21, pp. 328-336, 2008.

[12] D. Kaisarly and M. E. Gezawi, "Polymerization shrinkage assessment of dental resin composites: a literature review," Odontology, vol. 104, pp. 257-270, 2016.

[13] K. L. Van Landuyt, J. Snauwaert, J. De Munck et al., "Systematic review on the chemical composition of contemporary dental adhesives," Biomaterials, vol. 28, no. 26, pp. 3757-3785, 2007.

[14] R. R. Moraes, N. Boscato, P. S. Jardim, and L. F. J. Schneider, "Dual and self-curing potential of self-adhesive resin cements as thin films," Operative Dentistry, vol. 36, no. 6, pp. 635-642, 2011.

[15] V. Miletic, D. Manojlovic, M. Milosevic, N. Mitrovic, T. S. Stankovic, and T. Maneski, "Analysis of local shrinkage patterns of self-adhering and flowable composites using 3D digital image correlation," Quintessence. International, vol. 42, pp. 797-804, 2011.

[16] J. Li, A. S. L. Fok, J. Satterthwaite, and D. C. Watts, "Measurement of the full-field polymerization shrinkage and depth of cure of dental composites using digital image correlation," Dental materials, vol. 25, no. 5, pp. 582-588, 2009.

[17] L. C. Oliveira, S. Duarte, C. A. Araujo, and A. Abrahao, "Effect of low-elastic modulus liner and base as stress-absorbing layer in composite resin restorations," Dental materials, vol. 26, no. 3, pp. 159-169, 2010.

[18] C. A. Pulido, A. P. Gebert, F. de Oliveira et al., "An in situ evaluation of the polymerization shrinkage, degree of conversion, and bond strength of resin cements used for luting fiber posts," The Journal of Prosthetic Dentistry, vol. 116, no. 4, pp. 570-576, 2016.

[19] R. Jin-Young Kim, Y. Kim, N. Choi, and I. Lee, "Polymerization shrinkage, modulus, and shrinkage stress related to toothrestoration interfacial debonding in bulk-fill composites," Journal of Dentistry, vol. 43, no. 4, pp. 430-439, 2015.

[20] M. Martinsen, R. El-Hajjar, and D. W. Berzins, "3D full field strain analysis of polymerization shrinkage in a dental composite," Dental Materials, vol. 29, no. 8, pp. e161-e167, 2013.

[21] A. Lau, J. Li, Y. Cheul, C. Heo, and A. Fok, "A study of polymerization shrinkage kinetics using digital image correlation," Dental Materials, vol. 31, no. 4, pp. 391-398, 2015.

[22] V. Miletic, D. Peric, M. Milosevic, D. Manojlovic, and N. Mitrovic, "Local deformation fields and marginal integrity of sculptable bulk-fill, low-shrinkage and conventional composites," Dental Materials, vol. 32, no. 11, pp. 1441-1451, 2016.
[23] A. Mitrovic, I. Tanasic, N. Mitrovic, M. Milosevic, L. Tihacek-Sojic, and D. Antonovic, "Strain determination of self-adhesive resin cement using 3D digital image correlation method," Srpski Arhiv Za Celokupno Lekarstvo, vol. 146, no. 7-8, pp. 372-377, 2018.

[24] J. R. Bausch, K. de Lange, C. L. Davidson, A. Peters, and A. J. de Gee, "Clinical significance of polymerization shrinkage of composite resins," The Journal of Prosthetic Dentistry, vol. 48, no. 1, pp. 59-67, 1982.

[25] M. Tuntiprawon and P. R. Wilson, "The effect of cement thickness on the fracture strength of all-ceramic crowns," Australian Dental Journal, vol. 40, no. 1, pp. 17-21, 1995.

[26] P. Karntiang and C. Leevailoj, "Effect of Resin-Cement Thickness on Compressive-Fracture-Resistance of EnamelBonded Ceramic," CU Dent Journal, vol. 37, pp. 161-170, 2014.

[27] L. G. May, J. R. Kelly, M. A. Bottino, and T. Hill, "Effects of cement thickness and bonding on the failure loads of CAD/CAM ceramic crowns: multi-physics FEA modeling and monotonic testing," Dental Materials, vol. 28, no. 8, pp. e99-e109, 2012. 


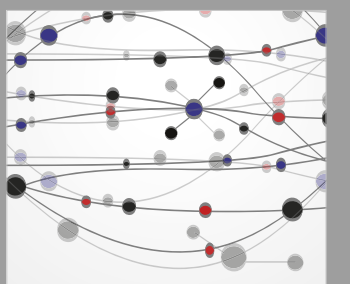

The Scientific World Journal
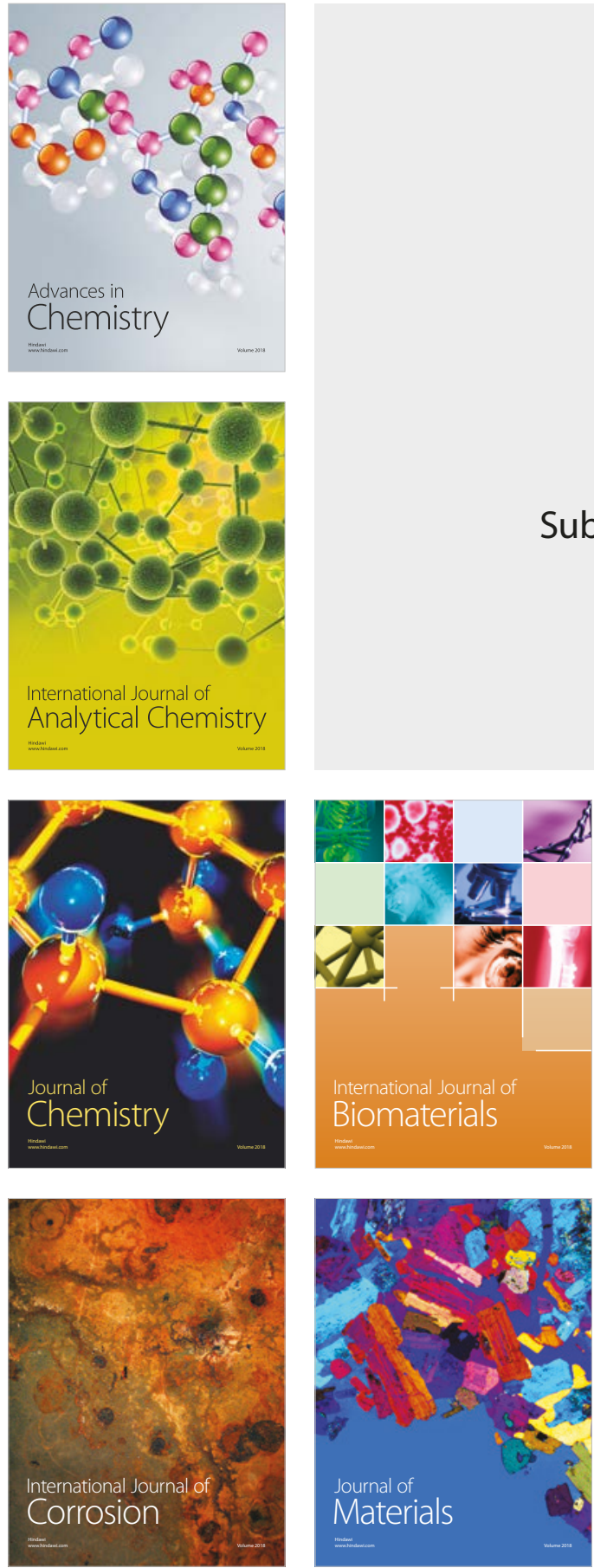

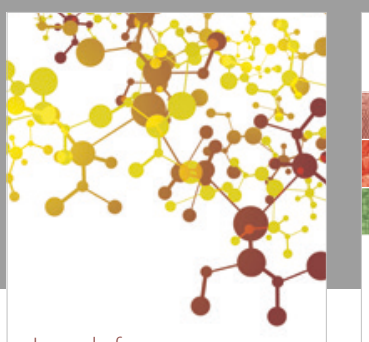

Journal of

Applied Chemistry
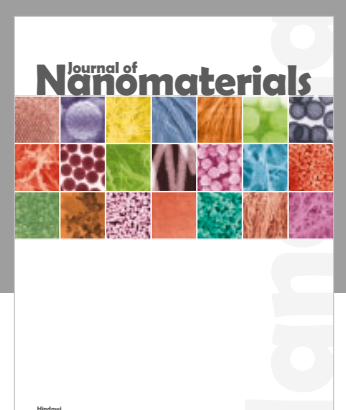

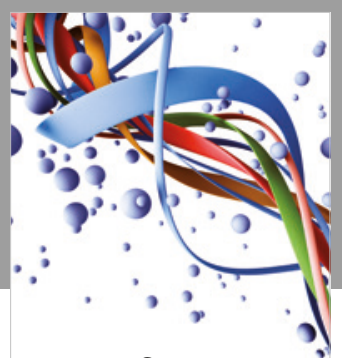

Scientifica

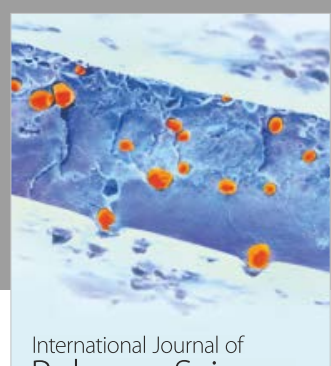

Polymer Science

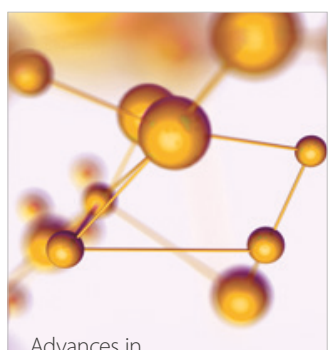

Physical Chemistry
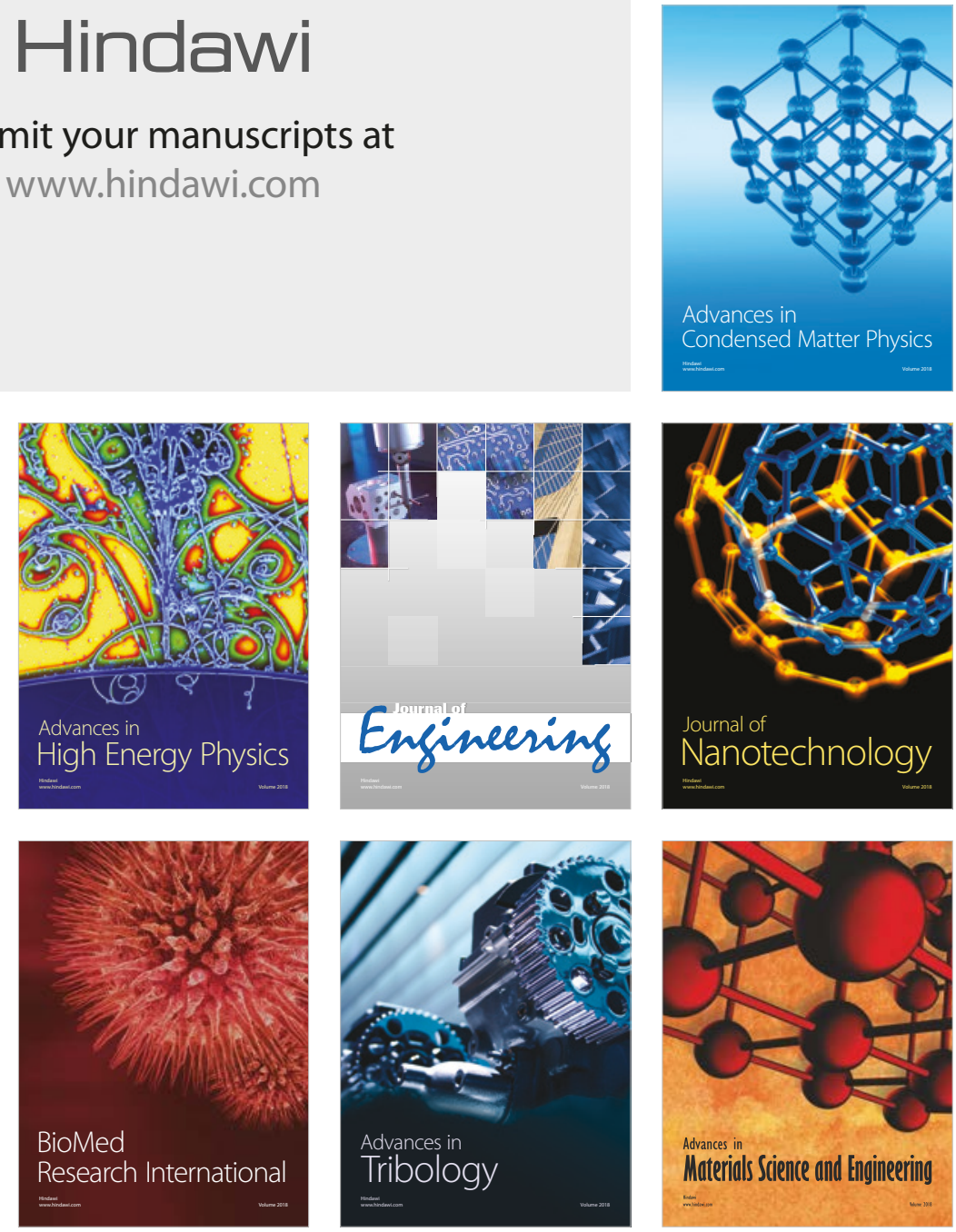